\title{
Agonist anti-GITR monoclonal antibody and stereotactic radiation induce immune-mediated survival advantage in murine intracranial glioma
}

\author{
Mira Patel ${ }^{1 *}$, Jennifer Kim², Debebe Theodros ${ }^{2}$, Christopher Jackson ${ }^{3}$, Ada Tam ${ }^{4}$, Esteban Velarde ${ }^{5}$, Betty Tyler ${ }^{3}$, \\ Xiaobu $\mathrm{Ye}^{3}$, Henry Brem ${ }^{3}$, Mark Selby ${ }^{6}$, Charles Drake ${ }^{7}$, Drew Pardoll ${ }^{1}$, Michael Lim $^{3}$ \\ From 30th Annual Meeting and Associated Programs of the Society for Immunotherapy of Cancer \\ (SITC 2015) \\ National Harbor, MD, USA. 4-8 November 2015
}

\section{Background}

Glioblastoma (GBM) is a poorly immunogenic neoplasm treated with local radiation. Despite the standard of care, median survival remains low. Immunotherapy has synergized with stereotactic radiosurgery (SRS) in murine GBM, as radiation promotes a pro-inflammatory tumor microenvironment amenable to the anti-tumor effects of immune modulation. Glucocorticoid-induced tumor necrosis factor receptor (GITR) is a co-stimulatory receptor expressed constitutively on regulatory $\mathrm{T}$ cells and inducibly on effector T cells. We tested the hypothesis that anti-GITR monoclonal antibody (mAb) and SRS combination therapy would confer immune-mediated survival benefit in murine glioma.

\section{Methods}

Mice were implanted with GL261-luc murine glioma cells and began SRS and anti-GITR IgG1 treatment after 10 days. Mice were randomized to four treatment groups: control, SRS only, anti-GITR only, anti-GITR+SRS. SRS was delivered to the tumor in one fraction; mice were given $\mathrm{mAb}$ thrice i.p. Mice were euthanized on day 21 to analyze the immunologic profile of tumor, spleen, and tumor draining lymph nodes.

\section{Results}

Anti-GITR mAb plus SRS conferred significantly improved survival over either treatment alone $(\mathrm{p}<.0001$, cure rate $24 \%$ ). The increased survival required $\mathrm{CD} 4+\mathrm{T}$ cells but not $\mathrm{CD} 8+\mathrm{T}$ cells or regulatory $\mathrm{T}$ cells (Tregs).

'Johns Hopkins University School of Medicine, Baltimore, MD, USA Full list of author information is available at the end of the article
There was elevated intratumoral CD4+ effector-cell infiltration (CD4+/Foxp3-/IFN $\gamma+$ ) relative to Treg infiltration $(\mathrm{CD} 4+$ /Foxp3+) at day 21 in mice treated with anti-GITR + SRS, and significantly elevated IFN $\gamma$ and IL-2 production by $\mathrm{CD} 4+\mathrm{T}$ cells and elevated IFN $\gamma$ and TNF $\alpha$ production by $\mathrm{CD} 8+\mathrm{T}$ cells. Intratumoral mononuclear cells demonstrated increased mRNA expression of pro-inflammatory M1 markers and decreased expression of immunosuppressive M2 markers.

\section{Conclusions}

In all, anti-GITR mAb synergizes with SRS to significantly prolong survival in murine orthotopic glioma in a potentially CD4+ Th1-dominant anti-tumor mechanism with M1 polarization. These findings provide preclinical evidence for the use of anti-GITR IgG1 non-depleting antibodies alongside SRS in human GBM.

\section{Authors' details \\ ${ }^{1}$ Johns Hopkins University School of Medicine, Baltimore, MD, USA. ${ }^{2}$ The Johns Hopkins University School of Medicine, Baltimore, MD, USA. ${ }^{3}$ The Johns Hopkins University Department of Neurosurgery, Baltimore, MD, USA. ${ }^{4}$ The Johns Hopkins University Department of Immunology, Baltimore, MD, USA. ${ }^{5}$ The Johns Hopkins University Department of Radiation Oncology, Baltimore, MD, USA. ${ }^{6}$ Bristol-Myers Squibb, Baltimore, MD, USA. ${ }^{7}$ The Johns Hopkins University Departments of Oncology and Cancer Immunology, Baltimore, MD, USA.}

Published: 4 November 2015

doi:10.1186/2051-1426-3-S2-P194

Cite this article as: Patel et al:: Agonist anti-GITR monoclonal antibody and stereotactic radiation induce immune-mediated survival advantage in murine intracranial glioma. Journal for ImmunoTherapy of Cancer 2015 3(Suppl 2):P194. 


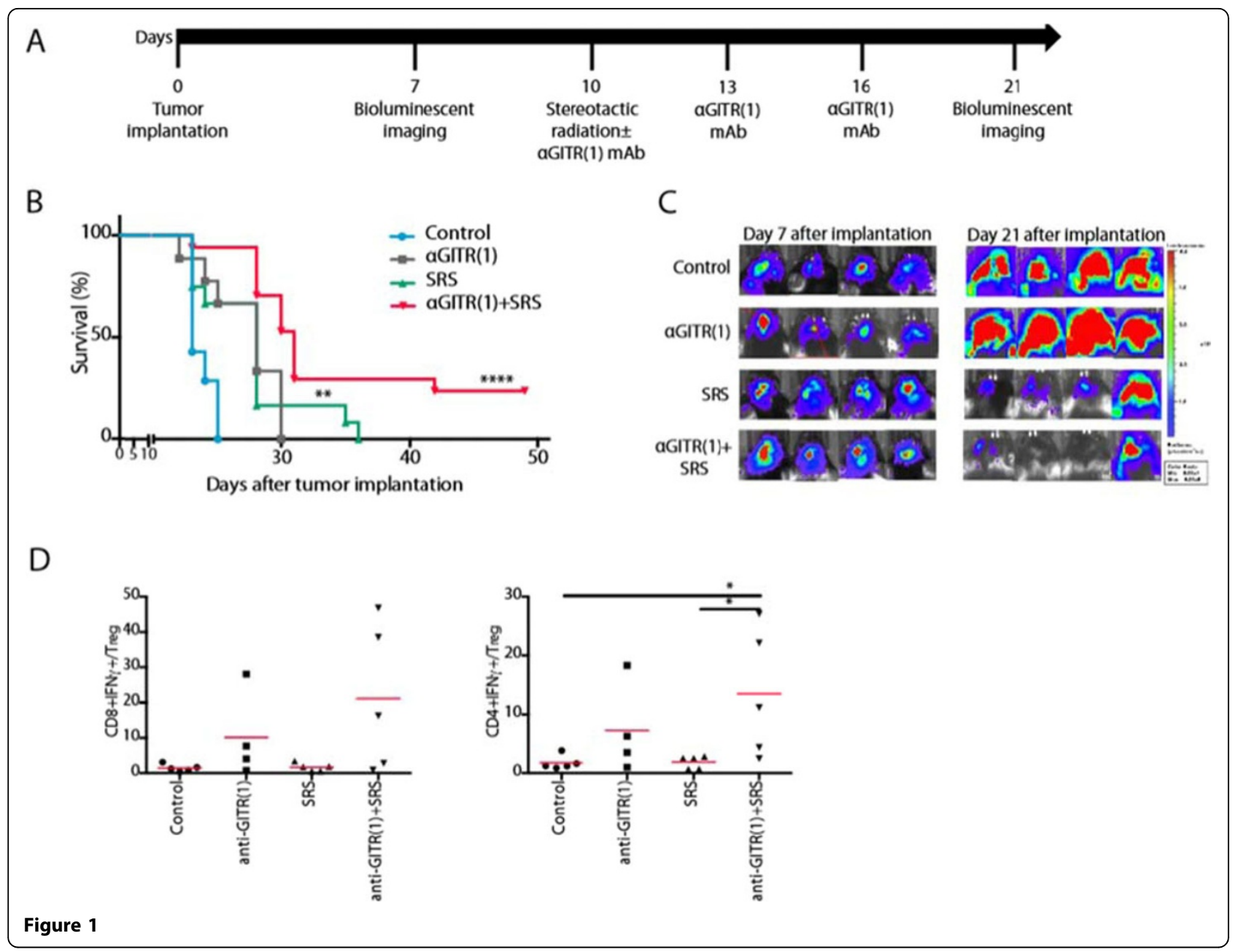

